\title{
Why should we focus on high-volume hemodiafiltration?
}

\author{
Sug-Kyun Shin ${ }^{1}$, Young-II Jo ${ }^{2}$ \\ ${ }^{1}$ Division of Nephrology, Department of Internal Medicine, NHIS Ilsan Hospital, Goyang, Republic of Korea \\ ${ }^{2}$ Division of Nephrology, Department of Internal Medicine, Konkuk University School of Medicine, Seoul, Republic of Korea
}

\begin{abstract}
Though noticeable technological advances related to hemodialysis (HD) have been made, unfortunately, the survival rate of dialysis patients has yet to improve significantly. However, recent research findings reveal that online hemodiafiltration (HDF) significantly improves patient survival in comparison to conventional HD. Accordingly, the number of patients receiving online HDF is increasing. Although the mechanism driving the benefit has not yet been fully elucidated, survival advantages are mainly related to the lowering of cardiovascular mortality. High cardiovascular mortality among HD patients is seemingly attributable to the cardiovascular changes that occur in response to renal dysfunction and the HD-induced myocardial stress and injury, and online HDF appears to improve such secondary cardiovascular changes. Interestingly, patient survival improves only if the convection volume is supplied sufficiently over a certain level during online HDF treatment. In other words, survival improvement from online HDF is related to convection volume. Therefore, there is a growing interest in high-volume HDF in terms of improving the survival rate. The survival improvement will require a minimum convection volume of $23 \mathrm{~L}$ or more per 4-hour session for postdilution HDF. To obtain an optimal high convection volume in online HDF, several factors, such as the treatment time, blood flow rate, filtration fraction, and dialyzer, need to be considered. High-volume HDF can be performed easily and safely in routine clinical practice. Therefore, when the required equipment is available, performing high-volume HDF will help to improve the survival rate of dialysis patients.
\end{abstract}

Keywords: Convective therapies, Chronic kidney failure, Hemodiafiltration, Mortality, Survival

\section{Introduction}

As underlying diseases of chronic kidney disease, such as diabetes, are surging drastically with the gradual increase in life expectancy, the number of patients with end-stage renal disease (ESRD) on dialysis is increasing explosively. According to Canaud et al.'s report [1] published in 2020, the number of patients with ESRD receiving renal replacement therapy reached 3,171,000 globally in 2017, among which those on hemodialysis (HD) accounted for
$2,823,000(89 \%)$. Further, of the 2,823,000 patients undergoing HD, 286,000 (10\%) were receiving hemodiafiltration (HDF). In 2009, the number of HDF patients was 77,300, increasing by 3.7 times to 286,000 in 2017 , resulting in an annual growth rate of as high as $18 \%$ [1]. With the explosive upsurge in the number of ESRD patients, significant advancements in dialysis therapy methods and technology have also occurred. However, despite such technological progress, patient survival has not improved much, and the lifespan of a dialysis patient remains shorter than that of

Received: November 14, 2021; Revised: December 20, 2021; Accepted: December 23, 2021

Correspondence: Young-II Jo

Division of Nephrology, Department of Internal Medicine, Konkuk University Medical Center, Konkuk University School of Medicine, 120-1

Neungdong-ro, Gwangjin-gu, Seoul 05030, Republic of Korea. E-mail: nephjo@kuh.ac.kr

ORCID: https://orcid.org/0000-0002-6695-7062

Copyright @ 2022 by The Korean Society of Nephrology

(a) This is an Open Access article distributed under the terms of the Creative Commons Attribution Non-Commercial and No Derivatives License (http:// creativecommons.org/licenses/by-nc-nd/4.0/) which permits unrestricted non-commercial use, distribution of the material without any modifications, and reproduction in any medium, provided the original works properly cited. 
nondialysis persons by more than 10 years. Moreover, the mortality rate of ESRD patients is 2.5 times higher than that of cancer patients [2].

Of interest, recent studies have reported that online HDF significantly improves patient survival in comparison to conventional HD. Irrespective of the mode of postdilution or predilution HDF, online HDF apparently helps to improve patient survival, as demonstrated by large-scale randomized controlled trials (RCTs) such as the CONTRAST (CONvective TRAnsport STtudy), the Turkish OL-HDF (Online Haemodiafiltration) trial, and the ESHOL (Estudio de Super-vivencia de Hemodiafiltración On-line) trial together with several meta-analyses considering these RCTs and pooled individual participant data (IPD) analyses [3-8]. In addition, evidence has suggested that in-center HDF is both more therapeutically effective and cost-effective than in-center HD. Based on these findings, the 2018 National Institute for Health and Care Excellence (NICE) guideline recommended that HDF should be considered rather than HD when dialysis is performed in-center [9]. Further, Maduell [3] proposed that adoption of HDF as a "conventional HD treatment" in ESRD patients should be considered, as it significantly improves survival rate compared to HD. Even before the NICE guideline was issued, research findings revealed that online HDF significantly improves patient survival compared to conventional HD, leading to a noticeable increase in the use of HDF in and around Europe and the Asia Pacific region. Given that patients with ESRD worldwide have increased in number at an annual growth rate of $18 \%$ from 2009 to 2017 and the annual growth rate of HD patients was only $6.6 \%$ during this period, such can be regarded as evidence of a noticeable increase in HDF patients [1]. Nevertheless, online HDF does not guarantee an unconditional increase in patient survival, as the survival rate is improved only when supplying a sufficient convection volume above a certain level, i.e., high-volume HDF $[8,10]$. In other words, a sufficient amount of convection volume during online HDF is required to improve the survival rate. Therefore, the Korean Society of Nephrology guideline for 2021 recommended that high-volume HDF be considered in consideration of its cost-effectiveness [11].

After the issuance of the NICE guideline in 2018, important new research findings were published revealing that high-volume HDF improves patient survival [12-16]. As improved survival rates among dialysis patients are a critical goal and a matter of concern, online HDF, particularly high-volume HDF, should be given special attention. Hence, this review will examine, through a literature analysis, the clinical benefit of high-volume HDF, the effect of improving the survival rate and its mechanism, and the method of implementing high-volume HDF.

\section{Survival improvement by high-volume hemodiafiltration}

Recent research findings have revealed that the survival improvement gained from online HDF is related to convection volume $[6,8,10,17,18]$. In other words, patient survival rates improve only if convection volume is supplied sufficiently above a certain level. CONTRAST was conducted involving patients of low-flux HD and postdilution HDF, and it reported no difference in all-cause mortality among these two groups. However, when patients were separated according to convection volume into three groups of $<18.17 \mathrm{~L}, 18.17$ to $21.95 \mathrm{~L}$, and $>21.95 \mathrm{~L}$, respectively, and subsequently analyzed, the $>21.95$-L group showed a significant improvement in survival (hazard ratio [HR], 0.57; $\mathrm{p}<0.02$ ) [4]. The Turkish OL-HDF study also reported this finding. In the Turkish OL-HDF study, while there existed no significant difference in death rate between patients who received high-flux HD and those who received postdilution HDF, the separation of HDF patients, based on a convection volume of $17.4 \mathrm{~L}$, and subsequent analysis demonstrated that, among the $>17.4$-L group, overall mortality and cardiovascular mortality rates decreased by $46 \%$ (relative risk [RR], 0.54; 95\% confidence interval [CI], 0.31-0.93; $\mathrm{p}=0.02$ ) and 71\% (RR, 0.29; 95\% CI, 0.12-0.65; $\mathrm{p}$ $=0.003$ ), respectively [5]. In the ESHOL study, an analysis of a limited group of high-efficiency postdilution HDF patients with convection volumes of 22.9 to $23.9 \mathrm{~L}$ documented an improvement in the all-cause mortality rate by $30 \%$ in comparison to HD patients $(\mathrm{p}=0.01)$ [6]. Peters et al. [8] conducted a pooled IPD analysis of 2,793 patients who participated in four well-known RCTs (CONTRAST, Turkish OL-HDF study, ESHOL, and FRENCHIE [French Convective versus Hemodialysis in Elderly] study) and revealed a survival improvement attributed to high-volume HDF. In other words, their IPD analysis indicated that, among online HDF patients, the overall mortality and cardiovascular mortality rates declined by $14 \%$ and $23 \%$, respectively. No- 
tably, the analysis of three groups ( $<19$ L, 19-23 L, and $>23$ L) of convection volume showed that survival remarkably improved (HR for all-cause mortality, 0.78; HR for cardiovascular mortality, 0.69 ) among the patients with convection volumes of $>23 \mathrm{~L}$ (per $1.73 \mathrm{~m}^{2}$ of body surface area per session) [8]. Nubé et al. [17] also reported in an IPD meta-analysis that high-volume HDF significantly reduced all-cause mortality and cardiovascular mortality; also, they found that, the higher the convection volume, the lower the risk of death. In 2019, a cohort study using data from the Australia and New Zealand Dialysis and Transplant Registry (ANZDATA) showed that survival was significantly higher under HDF than under HD [12].

These findings strongly imply that high-volume HDF is required to improve patient survival. Moreover, such improved survival from high-volume HDF was demonstrated not only in postdilution HDF but also in predilution HDF. In 2019, a study was conducted using data from the Japanese Society for Dialysis Therapy Renal Data Registry (JRDR) to analyze survival among HD and predilution HDF patients with 5,000 couples of a propensity-matched cohort, which produced the finding that predilution HDF led to significantly higher overall survival than HD (HR for all-cause mortality, 0.83). Notably, the patient group with a high substitution volume of $\geq 40$ L had significantly better survival than the patient group with a volume of $<40 \mathrm{~L}$. In the study, the optimal substitution volume for improved survival was 50.5 L (95\% CI, 39.0-63.5 L) [13]. Recently, Canaud et al. [18] reported that the convective dose for survival gain is a minimum of $75 \mathrm{~L}$ per week. All of these research findings point out that the most critical factor is not performing HDF but supplying enough convection volume during HDF. In short, volume is what matters.

\section{Survival improvement mechanism of high-volume hemodiafiltration}

Why is cardiovascular mortality high among hemodialysis patients?

Cardiovascular disease is the most common cause of death among conventional HD patients. According to the 2020 annual report of the United States Renal Data System (USRDS), cardiovascular disease accounts for $55.2 \%$ of deaths among ESRD patients receiving HD, and, in particular, cardiac arrhythmia/cardiac arrest accounts for $44.2 \%$ of allcause mortality [2]. Such a high cardiovascular mortality rate among HD patients is seemingly attributable to secondary cardiovascular changes due to renal dysfunction and HD-induced myocardial stress and injury. In ESRD patients, the secondary cardiovascular changes are caused by the multiple factors of fluid overload, uremic cardiomyopathy, secondary hyperparathyroidism, anemia, altered lipid metabolism, and accumulation of gut microbiota-derived uremic toxins like trimethylamine $\mathrm{N}$-oxidase; such cardiovascular changes contribute to a high number of deaths [19]. Nevertheless, cardiovascular mortality remains high even when these issues, such as fluid overload, hyperparathyroidism, and anemia, are dealt with, as HD itself apparently also causes myocardial stress and injury [20].

\section{Why do hemodiafiltration and hemodialysis differ in terms of mortality rate?}

While several studies have reported the finding that an appropriate increase in convection volume in online HDF enhances patient survival significantly, the mechanism by which high-volume HDF improves survival is still not fully understood [21]. However, considering the suggestion by previous studies that the observed survival advantages are mainly related to the reduction of cardiovascular mortality, the following potential mechanisms are suggested $[22,23]$.

\section{Intradialytic hemodynamic instability}

Intradialytic hypotension (IDH) is not uncommon during intermittent HD. Locatelli et al. [24] reported that the incidence of symptomatic IDH among HD patients was $7.5 \%$ across 28,950 sessions. During dialysis, fluid removal occurs in the intravascular compartment, and removed fluid is replenished from the interstitial fluid compartment. Mismatching between the refill rate and the plasma-removal rate creates volume contraction, which, for a normal person, engages the baroreceptor-mediated reflex to function and does not cause hypotension. However, in a uremic patient, mismatching-induced volume contraction hinders the baroreceptor-mediated reflex, finally resulting in IDH. Subsequently, IDH induces myocardial stress, which ultimately contributes to a high mortality rate among dialysis patients. In contrast, HDF reduces the frequency of IDH development significantly; in other words, HDF improves 
intradialytic hemodynamic instability [17]. Locatelli et al. [24] reported that carrying out HDF reduced the frequency of symptomatic IDH onset by $50.9 \%$. In the FRENCHIE study, which was conducted among elderly patients to study intradialytic treatment tolerance, the frequencies of the intradialytic symptomatic hypotension development for HD and HDF recipients were $1.73 \%$ and $1.39 \%$, respectively, being significantly lower among HDF patients [25]. Nubé et al. [17] determined during an IPD meta-analysis using data of large-scale RCTs that the survival improvement gained from HDF is attributable to a reduction in fatal cardiac events, which is related to the improvement of intradialytic hemodynamic instability. Though administering replacement solution in large volumes may raise concerns about a potential risk of fluid overload during high-volume HDF, Chazot et al. [26] reported that there existed no significant difference in fluid overload development between HD and HDF patients. The cooling effect of replacement solution in large volumes also helps maintain hemodynamic stability [27].

\section{Endothelial dysfunction}

There is some evidence that HDF improves endothelial dysfunction. An RCT of $42 \mathrm{HD}$ patients measured flow-mediated dilatation and carotid distensibility after carrying out HD and high-volume $\mathrm{HDF}$ ( $>22 \mathrm{~L} /$ session) for 4 months, which led to significant improvement only in HDF patients. Such improvement in endothelial dysfunction was associated with oxidative stress, inflammation, and nitric oxide [28]. In the HDF, heart, and height $(3 \mathrm{H})$ study of pediatric patients, markers of endothelial dysfunction, such as asymmetric dimethylarginine (ADMA) and symmetric dimethylarginine (SDMA), improved significantly during HDF treatment $[29,30]$.

\section{Inflammation/oxidative stress}

There are several reports that suggest HDF can help to improve inflammation and oxidative stress. A follow-up study of CONTRAST traced the changes in C-reactive protein (CRP) level for 3 years and found that, despite increasing significantly among HD patients, the CRP level remained stable among HDF patients [31]. The $3 \mathrm{H}$ study revealed that the total antioxidant capacity improved during HDF treatment, while inflammation markers such as high-sensitivity CRP significantly decreased [30].

\section{Vascular stiffness}

Pulse-wave velocity (PWV) is a surrogate measurement that indicates vascular stiffness. Despite increasing significantly within 6 months after the initiation of HD, aortic PWV remained stable in HDF patients. Also, the diastolic relaxation area, which indicates left ventricular refilling, increased significantly among HDF patients [32]. In another RCT that followed patients for 1 year after treatment, brachial PWV increased significantly after HD onset while remaining stable among HDF patients [33].

\section{Cardiac remodeling}

HDF significantly reduces the levels of nitriuretic peptides, such as brain natriuretic peptide (BNP), N-terminal-proBNP (NT-proBNP), and proBNP. These nitriuretic peptides are significantly correlated with certain echocardiography remodeling parameters, such as left atrium diameter, left ventricular diastolic diameter, and left ventricular mass index (LVMI), and HDF reduces such a cardiac burden [34]. Another prospective crossover study also demonstrated that, while there were no significant changes in the left atrium diameter under HD, it declined significantly under HDF, which was correlated with the decrease in nitric oxide and ADMA [35]. Recently, Páll et al. [36] reported that $\mathrm{T}$ peak-end interval was lower under HDF than under HD and showed a significant correlation with LVMI. However, in a recently released meta-analysis, conventional HD and HDF showed no significant variations in the impact on the changes of LVMI and ejection fraction [37]. Hence, additional studies will be required to demonstrate whether HDF helps improve left ventricular hypertrophy to enhance survival.

\section{Sympathetic tone activity}

Chronic sympathetic overactivity is a well-known cardiovascular risk factor. In a prospective study, Park et al. [38] reported that online HDF improved autonomic nervous system dysfunction in chronic dialysis patients.

\section{How to perform high-volume hemodiafiltration}

\section{What is the optimal convection volume?}

Although the mechanism has not been fully elucidated, it is apparently obvious that high-volume HDF helps to im- 
prove patient survival. Nonetheless, there still remains a question of whether any selection bias has played a role in improving the survival from high-volume HDF in previous studies $[16,39]$. For example, in their pooled IPD analysis, Peters et al. [8] carried out online HDF with the convective dose of 69 L per week or higher, which requires patients to have vascular access in good condition. In other words, it cannot be completely ruled out that study participants might have included only those healthy enough to endure such a high dose. Despite these limitations, however, all of these studies support the conclusion that high-volume HDF improves survival significantly in a manner largely attributable to reduced cardiovascular mortality.

There have been several rounds of discussion about how much of a convection volume is required at minimum to gain improved survival. Previous reports have indicated that the volume threshold for improved survival varied between studies, ranging from a minimum of $15 \mathrm{~L}$ to a maximum of 23.1 L [40]. This phenomenon is due to differing study schemes and volume definitions. Peters et al. [8] reported in their pooled IPD analysis that survival improvement required a minimum convection volume of $23 \mathrm{~L} / 1.73$ $\mathrm{m}^{2}$ body surface area per HDF session. Previous research findings suggest that, for postdilution HDF, the survival improvement will require a minimum convection volume of $\geq 23$ L per 4-hour session [41-44]. Based on the results of the main clinical trials, the current recommendation for high-volume HDF on a thrice-weekly treatment schedule would be a convective volume of $>23 \mathrm{~L} /$ session [44]. Considering the dilution factor, a minimum convection volume of $46 \mathrm{~L}$ per session would be required for predilution HDF [43,45]. According to a report using data from the JRDR database, the optimal substitution volume leading to improved survival was estimated to be $50.6 \mathrm{~L} /$ session in patients receiving predilution HDF [13]. Therefore, more important than anything else is carrying out high-volume HDF therapy that supplies convection volume at a sufficient level of $\geq 23 \mathrm{~L} /$ session, not just carrying out HDF $[8,12,13]$.

What are the factors for the optimal level of high convection volume?

As reviewed so far, determining how to obtain a high convection volume of $\geq 23 \mathrm{~L} /$ session is a key to conducting
HDF successfully. However, there is an issue that high convection volumes may not be obtained automatically just by performing online HDF treatment. In CONTRAST, for example, only $22 \%$ of participating patients reached a target convection volume of $24 \mathrm{~L}$ [4], which points out that a special strategy should be deployed to achieve high convection volumes. Several factors, such as treatment time, blood flow rate, filtration fraction, and dialyzer, are required to obtain an optimal high convection volume in online HDF [40,46-48]. Below are the respective reviews of these factors.

\section{Treatment time}

Treatment time is a crucial determining factor of convection volume, and one of the surest ways to increase the convection volume is by extending the treatment time [46], which will increase the convection volume with ease. With a blood flow rate of $400 \mathrm{~mL} / \mathrm{min}$ and a filtration fraction of $25 \%$ in postdilution HDF, extending the treatment time by one hour can increase the convection volume by approximately $6 \mathrm{~L}$ [48]. Therefore, if blood flow is insufficient, it is advisable to increase the treatment time to obtain a high convection volume. However, extending the treatment time by one hour is not an easy task in the real world. Extending the treatment time even 30 minutes, if not one hour, would help to increase the convection volume. In CONTRAST, a 30-minute extension of the treatment time increased convection volume by approximately $2.5 \mathrm{~L}$ [46].

\section{Blood flow rate}

Blood flow rate is also a critical determinant of convection volume, and it represents the most important limiting factor, especially in postdilution $\operatorname{HDF}[3,49,50]$. In postdilution HDF, to reduce the risk of increasing viscosity and clotting complications, a dialyzer reduces the infusion flow to $\leq 33 \%$ of the blood pump flow rate $(\mathrm{Qb})$. Therefore, it is recommended that, in postdilution $\mathrm{HDF}$, the blood flow rate should be kept at 350 to $500 \mathrm{~mL} / \mathrm{min}$ to obtain a sufficiently high convection volume $[3,22,40,51]$. If carrying out postdilution HDF is unlikely to deliver this level of blood flow rate, it may prevent the expected clinical benefits from online HDF from manifesting. If this level of blood flow rate is hard to come by, predilution HDF offers a better option $[52,53]$. Predilution HDF makes it possible to obtain a high convection volume with a low blood flow rate of 200 to 250 
$\mathrm{mL} / \mathrm{min}$ [13].

On the other hand, if a blood flow rate may not reach 350 to $500 \mathrm{~mL} / \mathrm{min}$ in postdilution $\mathrm{HDF}$, a high convection volume can be obtained by increasing the treatment time, adjusting the filtration fraction, and using an auto-substitution mode $[47,49,54,55]$. Nevertheless, the blood flow rate is still very important. Even if a recommended level of 350 to $500 \mathrm{~mL} / \mathrm{min}$ is not reached, the higher the blood flow rate, the easier it gets to carry out high-volume HDF. Maduell et al. [49] studied the influence of blood flow rate on convection volume by adjusting the blood flow rate to 250,300 , 350,400 , and $450 \mathrm{~mL} / \mathrm{min}$, respectively, per session in 23 patients undergoing postdilution HDF. The convection volumes in each session were $23.7 \pm 2.2,26.9 \pm 3.1,30.2 \pm 2.3$, $32.8 \pm 3.3$, and $35.2 \pm 2.9 \mathrm{~mL} / \mathrm{min}$, respectively, and higher blood flow rates led to increased levels of convection volume; specifically, the convection volume increased by 8 to $12 \mathrm{~mL} / \mathrm{min}$ for each surge of blood flow rate by $50 \mathrm{~mL} / \mathrm{min}$ [49]. Therefore, maintaining the blood flow rate at a high level, if possible, is critical to obtaining enough convection volume.

Two key determining factors of the blood flow rate are the type of vascular access and needle size [47]. If there are no problems, such as stenosis, an arteriovenous fistula (AVF) or arteriovenous graft (AVG) may generally achieve a blood flow rate higher than that achieved with a central venous catheter (CVC). In a study conducted by Marcelli et al. [50] of 3,315 postdilution HDF patients in six European countries, the blood flow rates of AVF, AVG, and CVC were $391 \pm 64,390 \pm 62$, and $316 \pm 50 \mathrm{~mL} / \mathrm{min}$, respectively. Considering the degree of reaching a substitution volume of $\geq 21 \mathrm{~L}$ in a multivariate logistic regression analysis, AVG was lower than AVF by $41.5 \%$ and CVC was lower than AVF by $57.8 \%$ [50]. However, having CVC does not necessarily mean there is an inability to conduct high-volume HDF [56]. In one study that analyzed CONTRAST data, which compared convection volume in patients undergoing postdilution HDF with AVF, AVG, or CVC, the convection volumes were $19.7 \pm 4.4,19.3 \pm 4.4$, and $21.9 \pm 4.4 \mathrm{~mL} / \mathrm{ses}-$ sion, respectively, with no difference between the types of vascular access [46].

For patients with AVF or AVG, needle size is important to achieve a high blood flow rate. If not for the issue of stenosis, a large needle would help to increase the blood flow rate. There is a general concern that the use of a large needle exacerbates the shunt outcome. However, according to one observational study, there existed no difference in complications between 14-, 15-, and 16-gauge needles [57]. Therefore, an insufficient level of convection volume will necessitate the use of a larger-sized needle. It is recommended to use a 16-gauge needle for a blood flow rate of 300 to $350 \mathrm{~mL} / \mathrm{min}$ and a 15-gauge needle for a blood flow rate of 350 to $400 \mathrm{~mL} / \mathrm{min}[47,58]$. In a study conducted in Europe, $61.3 \%$ of the needles used were 15-gauge, and approximately $85 \%$ of patients had a blood flow rate of $\geq 300$ $\mathrm{mL} / \mathrm{min}$ [59]. Another study indicated that a needle larger than 15-gauge was not needed to carry out high-volume HDF [55]. It should be noted that an increase in the blood flow rate may lead to an increase in the recirculation rate [60], which also further increases with inappropriate arterial inflow due to lowered cardiac output or stenosis in the venous outflow. As a higher recirculation rate may hinder efforts to obtain a sufficient level of convection volume, it is necessary to monitor the recirculation rate regularly [47].

\section{Filtration fraction}

Filtration fraction is also an important determining factor for convection volume. In clinical practice, the filtration fraction is defined as "a ratio of the convection volume to the blood flow rate." Accordingly, a higher filtration fraction heightens ultrafiltration, leading to an elevated convection volume. However, in postdilution HDF, replacement solution is injected after the dialyzer, increasing the hemoconcentration in the dialyzer, which causes coagulation in the dialyzer and damages it. Accordingly, the European Dialysis Working Group recommended a filtration traction of $20 \%$ to $25 \%$ as appropriate in postdilution HDF [61]. Nevertheless, the use of a dialysis machine with an automatic pressure-control mode may extend the filtration fraction up to $30 \%$ [62]. The use of a dialysis machine with an automatic pressure-control mode can keep the transmembrane pressure within a range of 180 to $190 \mathrm{mmHg}$ to prevent hemoconcentration, helping to obtain a high convection volume [49]. Meanwhile, an appropriate anticoagulation therapy is essential as a higher filtration fraction elevates blood coagulation in the dialyzer due to hemoconcentration. The convective clearance of heparin when used as an anticoagulant (unfractionated heparin or low-molecular-weight heparin) increases with the surge of the convection volume in online HDF. Therefore, the dose of heparin 
in HDF may be $10 \%$ higher than in HD [5].

\section{Dialyzer}

Online HDF necessitates a highly permeable membrane to filter water and middle-molecule solutes, and the membrane must be properly sized for the blood flow rate [51] that is, a high-flux membrane should have an ultrafiltration coefficient (KUF) of $>20 \mathrm{~mL} / \mathrm{hr} / \mathrm{mmHg} / \mathrm{m}^{2}$ and a sieving coefficient of $>0.6$ for $\beta_{2}$-microglobulin [43]. The membrane size should be at least 0.80 to $1.0 \mathrm{~m}^{2}$ per $200 \mathrm{~mL} / \mathrm{min}$ for effective extracorporeal blood flow, meaning that a 2.0$\mathrm{m}^{2}$-sized hemodialyzer is appropriate for a blood flow rate of $400 \mathrm{~mL} / \mathrm{min}$ [63].

Strategies to obtain an optimal convection volume for high-volume hemodiafiltration in the real world

In 2015, Chapdelaine et al. [47] suggested a method for obtaining an appropriate level of convection volume, which reported that a stepwise increase of the factors of treatment time, blood flow rate, and filtration fraction helps to increase the convection volume. In other words, for a patient with a blood flow rate of $300 \mathrm{~mL} / \mathrm{min}$, filtration fraction of $25 \%$, and treatment time of 210 minutes, the convection volume was $15.8 \mathrm{~L} /$ session; with the extension of treatment time to 240 minutes, the convection volume increased to $18.0 \mathrm{~L} /$ session. At this time, when the blood flow rate was increased from 300 to $350 \mathrm{~mL} / \mathrm{min}$, the convection volume increased from 18.0 to $21.0 \mathrm{~L} /$ session. Further, an increase in the blood flow rate to $400 \mathrm{~mL} / \mathrm{min}$ caused the convection volume to increase from 21.0 to $24.0 \mathrm{~L} / \mathrm{session}$. Stepwise adjustment of treatment time, blood flow rate, and filtration fraction in this patient allegedly led the convection volume to finally rise up to $29.8 \mathrm{~L}$ [47]. de Roij van Zuijdewijn et al. [54] conducted a prospective multicenter study to determine how many postdilution HDF patients would show a convection volume of $\geq 22$ L would be obtainable when such a stepwise protocol is applied. This study applied the stepwise protocol in all patients to increase the treatment time to 4 hours, the blood flow rate to $400 \mathrm{~mL} /$ min, and the filtration fraction to $33 \%$ if possible, which produced the result that a convection volume of $\geq 22 \mathrm{~L}$ was achieved in more than $80 \%$ of patients with an average of $26 \mathrm{~L} /$ session. A high convection volume could be obtained by increasing the blood flow rate and filtration fraction even when the treatment time could not be extended [54].

In actual clinical practice, it is not difficult to obtain a high convection volume if the treatment time, blood flow rate, and filtration fraction were adjusted appropriately according to patient condition by referring to the above stepwise protocol suggested by Chapdelaine et al. [47]. For a low blood flow rate, the switch from postdilution HDF to predilution HDF will help to increase in convection volume. Jo et al. [64] reported that this switch could achieve a high convection volume in $>90 \%$ of patients. Taking an actual example, convection volume for a female patient aged 74 years under postdilution HDF was $24.0 \mathrm{~L}$, but it reached 30.2 L after applying the stepwise protocol [64] (Fig. 1). Recently, Kim et al. [65] reported in a prospective observational study that a sufficiently high convection volume could be obtained by appropriately adjusting the blood flow rate, needle size, and dialyzer surface area. In this prospective observational study, an 8-step stepwise protocol was applied to 30 patients receiving postdilution HDF. The researchers first gradually increased the blood flow rate $(280 \rightarrow 300 \rightarrow 330 \mathrm{~mL} / \mathrm{min}$, steps $1-3)$, followed by needle size ( $16 \rightarrow 15$ gauge, step 4$)$, and the dialyzer surface area $\left(1.8 \rightarrow 2.5 \mathrm{~m}^{2}\right)$ was increased sequentially. After changing the dialyzer surface area, the blood flow rate and needle size were increased sequentially in the same manner (steps $5-8$ ). In step 1, 13.3\% of patients reached a substitution volume of $\geq 21 \mathrm{~L}$, while $96.7 \%$ of patients achieved a high convection volume after step 8 [65]. In conclusion, it is not difficult to obtain an appropriate level of convection volume for high-volume HDF in the real world by increasing the treatment time, blood flow rate, needle size, dialyzer surface area, and filtration fraction using a stepwise protocol. The recommended adequate prescriptions and requirements for successful high-volume HDF are summarized in Table $1[43,51]$.

\section{Points to consider when interpreting the results of studies reported to date on hemodiafiltration}

There are discrepancies among existing studies on whether high-volume HDF reduces the risk of mortality in patients with ESRD. Furthermore, the quality of evidence derived from the RCTs reported to date is considered low due to methodological limitations-that is, most trials were not specifically designed to evaluate the effects of different 


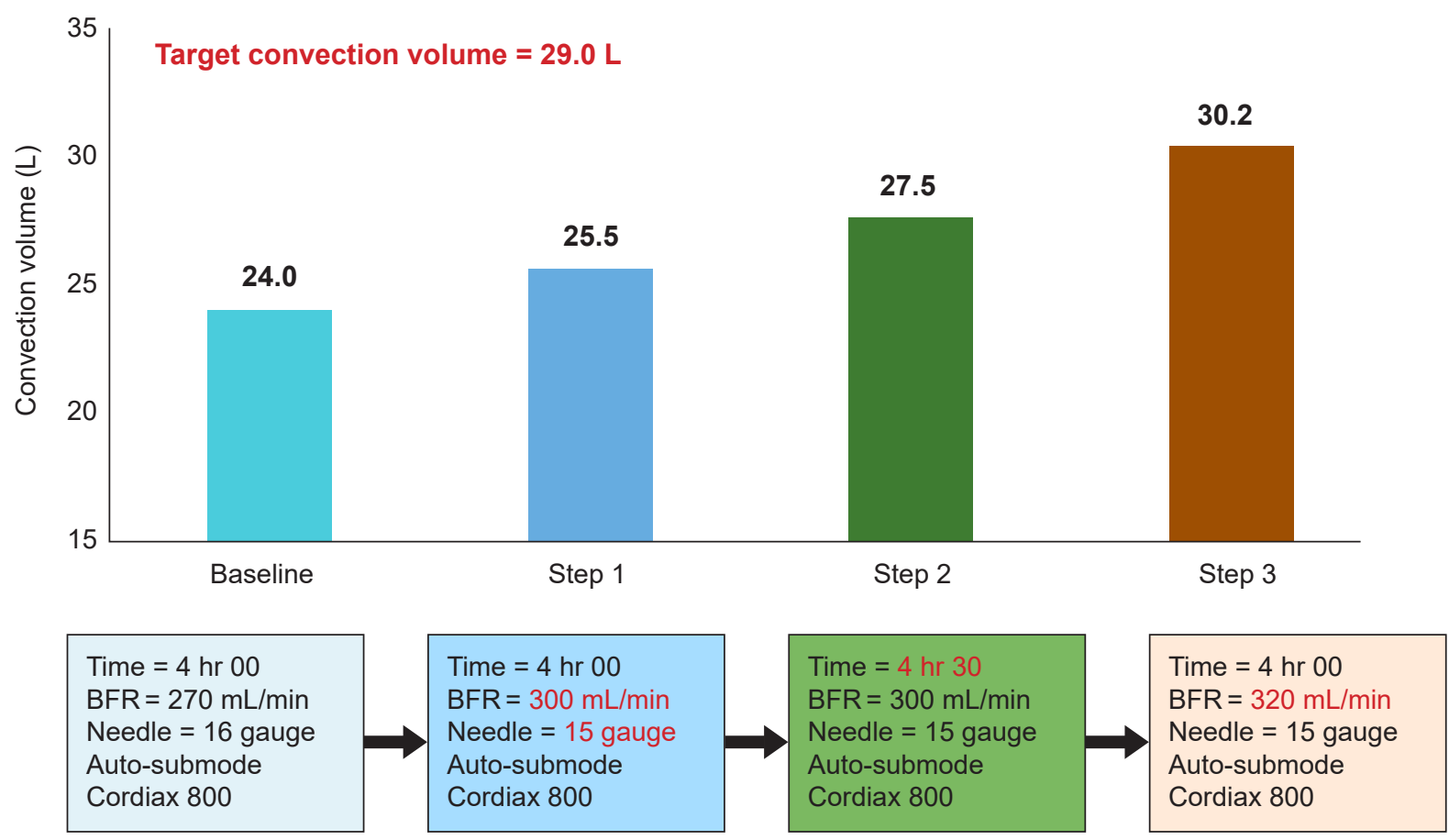

Figure 1. Case of applying a stepwise protocol to obtain the convection volume in the real world. A 74-year-old female patient with a height of $164 \mathrm{~cm}$ and a weight of $114 \mathrm{~kg}$ underwent postdilution hemodiafiltration (HDF). Initially, the treatment time was 4 hours, and the blood flow rate (BFR) was $270 \mathrm{~mL} / \mathrm{min}$. An increase in the BFR to $300 \mathrm{~mL} / \mathrm{min}$ led the convection volume to increase to 25.5 $\mathrm{L}$. The treatment time was extended to 4 hours and 30 minutes when the target convection volume of $29 \mathrm{~L} / \mathrm{session}$ was not reached, even after maximizing the filtration fraction via an auto-substitution (auto-sub) mode. However, the convection volume did not reach the target at $27.5 \mathrm{~L}$. Finally, when the BFR was further increased to $320 \mathrm{~mL} / \mathrm{min}$, the convection volume reached $30.2 \mathrm{~L}$.

Table 1. Prerequisites for successful high-volume HDF

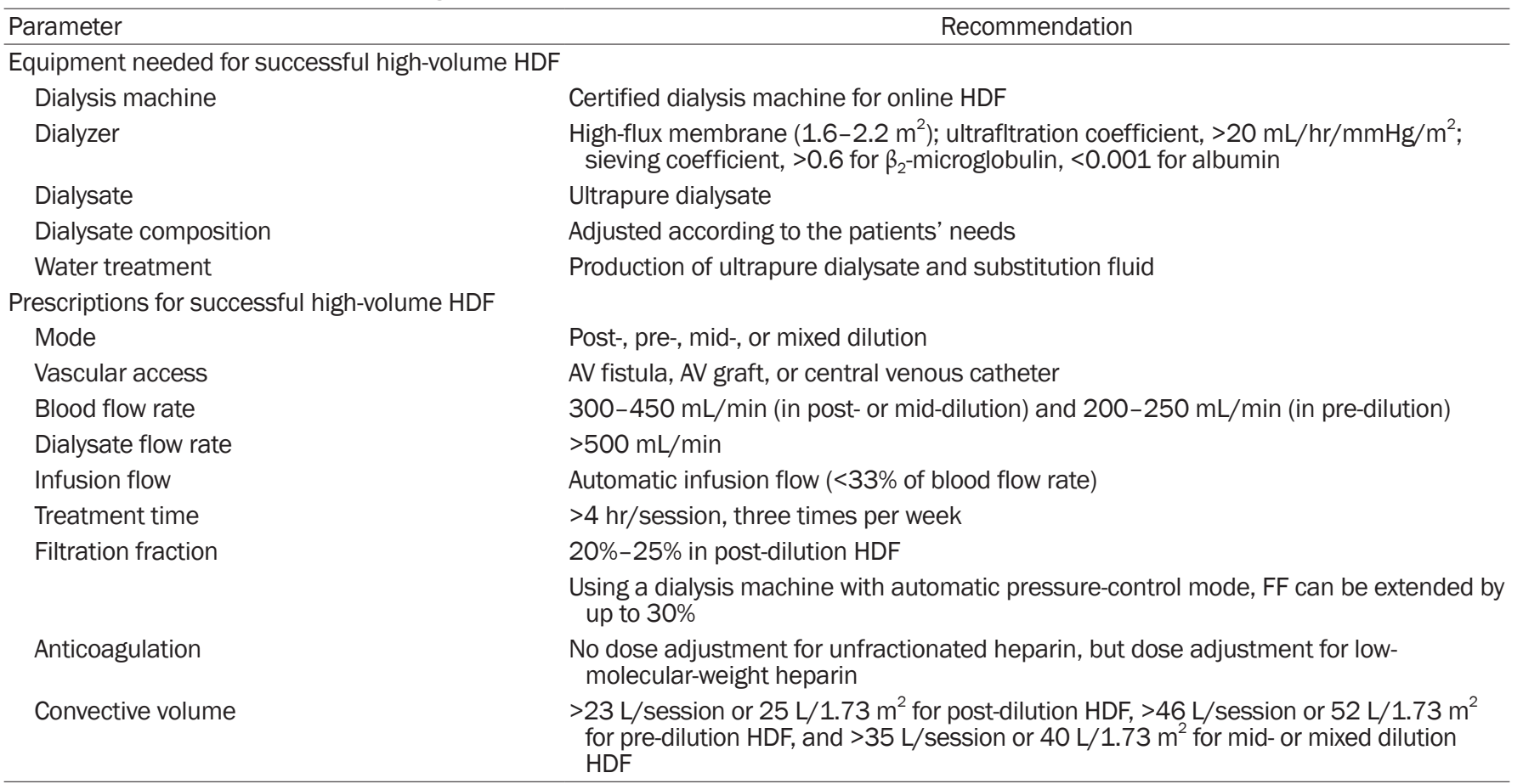

$\mathrm{AV}$, arteriovenous; FF, filtration fraction; HDF, hemodiafiltration. 
convective volumes. In addition, there was significant heterogeneity in dialysis interventions, including convective modalities, among trials. Therefore, caution is required when evaluating the effect of high-volume HDF on patient mortality.

In particular, the possibility that selection bias may have affected mortality in each study should be considered. High-volume postdilution HDF requires a higher blood flow rate, and high blood flow rates are better reached in patients with good vascular access, who also tend to be healthier individuals. Ultimately, this selection bias may affect patient mortality. If the patients selected for RCT were healthier than those excluded from the study, there is no doubt that the selection bias could affect study outcomes and lead to a lower all-cause mortality rate [66]. Indeed, compared to the DOPPS (Dialysis Outcomes and Practice Patterns Study) HDF population, HDF patients who participated in the three large studies (CONTRAST, Turkish OL-HDF study, and ESHOL) had well-functioning vascular access $[4,5,6,16]$. However, in a Japanese study using the JRDR database, blood flow rates of patients receiving predilution HDF were not higher than those with HD ( $p=0.99$ ) after propensity score matching. Nevertheless, patients undergoing high-volume predilution HDF had a significantly lower mortality rate than those receiving HD or low-volume predilution HDF [13].

Although high-volume HDF has shown several promising survival advantages over conventional HD, it remains controversial whether conclusive evidence is sufficient. Therefore, definitive studies are needed to determine whether high-volume HDF is preferred over standard highflux HD. For this purpose, the CONVINCE (comparison of high-dose HDF with high-flux HD) study, an international, multicenter, prospective, open-label RCT, is currently in progress to compare the benefits and harms of high-volume HDF and high-flux HD [67]. It is expected that this study will provide conclusive evidence of the superiority of high-volume HDF over standard high-flux HD in terms of the impact on all-cause mortality.

\section{Conclusion}

Although all data to date do not yet provide an accurate account of the exact mechanism, high-volume HDF apparently does provide a significant survival benefit compared to conventional HD. On the other hand, compared to conventional $\mathrm{HD}$, high-volume HDF led to almost no issues when carried out, and an appropriate level of convection volume is easily obtainable in routine clinical practice. Therefore, when the required equipment is available, performing high-volume HDF can help to improve the survival rate of dialysis patients.

\section{Conflicts of interest}

All authors have no conflicts of interest to declare.

\section{Authors' contributions}

Conceptualization, Data curation, Visualization: YIJ Investigation: SKS

Formal analysis, Methodology, Project administration: SKS, YIJ

Writing-original draft: SKS, YIJ

Writing-review \& editing: YIJ

All authors read and approved the final manuscript.

\section{ORCID}

Sug-Kyun Shin, https://orcid.org/0000-0001-7866-0955

Young-Il Jo, https://orcid.org/0000-0002-6695-7062

\section{References}

1. Canaud B, Köhler K, Sichart JM, Möller S. Global prevalent use, trends and practices in haemodiafiltration. Nephrol Dial Transplant 2020;35:398-407.

2. United States Renal Data System. 2020 USRDS annual data report: epidemiology of kidney disease in the United States [Internet]. Bethesda, MD: National Institutes of Health, National Institute of Diabetes and Digestive and Kidney Diseases; c2020 [cited 2021 Nov 14]. Available from: https://adr.usrds.org/2020/ end-stage-renal-disease/5-mortality.

3. Maduell F. Hemodiafiltration versus conventional hemodialysis: should "conventional" be redefined? Semin Dial 2018;31:625632.

4. Grooteman MP, van den Dorpel MA, Bots ML, et al. Effect of online hemodiafiltration on all-cause mortality and cardiovascular outcomes. J Am Soc Nephrol 2012;23:1087-1096.

5. Ok E, Asci G, Toz H, et al. Mortality and cardiovascular events in 
online haemodiafiltration (OL-HDF) compared with high-flux dialysis: results from the Turkish OL-HDF Study. Nephrol Dial Transplant 2013;28:192-202.

6. Maduell F, Moreso F, Pons M, et al. High-efficiency postdilution online hemodiafiltration reduces all-cause mortality in hemodialysis patients. J Am Soc Nephrol 2013;24:487-497.

7. Mostovaya IM, Blankestijn PJ, Bots ML, et al. Clinical evidence on hemodiafiltration: a systematic review and a meta-analysis. Semin Dial 2014;27:119-127.

8. Peters SA, Bots ML, Canaud B, et al. Haemodiafiltration and mortality in end-stage kidney disease patients: a pooled individual participant data analysis from four randomized controlled trials. Nephrol Dial Transplant 2016;31:978-984.

9. National Institute for Health and Care Excellence (NICE). NICE guideline (NG107): renal replacement therapy and conservative management [Internet]. London: NICE; c2018 [cited 2021 Nov 14]. Available from: http://www.nice.org.uk/guidance/ng107.

10. Davenport A, Peters SA, Bots ML, et al. Higher convection volume exchange with online hemodiafiltration is associated with survival advantage for dialysis patients: the effect of adjustment for body size. Kidney Int 2016;89:193-199.

11. Korean Society of Nephrology (KSN). KSN guideline 2021: evidence-based practice guidelines for appropriate hemodialysis treatment [Internet]. Seoul (Korea): KSN; c2021 [cited 2021 Nov 14]. Available from: http://www.ksn.or.kr/bbs/?code=g_guideline.

12. See EJ, Hedley J, Agar JW, et al. Patient survival on haemodiafiltration and haemodialysis: a cohort study using the Australia and New Zealand Dialysis and Transplant Registry. Nephrol Dial Transplant 2019;34:326-338.

13. Kikuchi K, Hamano T, Wada A, Nakai S, Masakane I. Predilution online hemodiafiltration is associated with improved survival compared with hemodialysis. Kidney Int 2019;95:929-938.

14. Neri L, Gurevich K, Zarya Y, et al. Practice patterns and outcomes of online hemodiafiltration: a real-world evidence study in a Russian Dialysis Network. Blood Purif 2021;50:309-318.

15. Tiranathanagul K, Susantitaphong P, Srisawat N, et al. Ten-year survival of end-stage renal disease patients treated with high-efficiency online hemodiafiltration: a cohort study of a center in South East Asia. Nephron 2018;139:150-158.

16. Locatelli F, Karaboyas A, Pisoni RL, et al. Mortality risk in patients on hemodiafiltration versus hemodialysis: a 'real-world' comparison from the DOPPS. Nephrol Dial Transplant 2018;33:683689.

17. Nubé MJ, Peters SA, Blankestijn PJ, et al. Mortality reduction by post-dilution online-haemodiafiltration: a cause-specific analysis. Nephrol Dial Transplant 2017;32:548-555.

18. Canaud B, Barbieri C, Marcelli D, et al. Optimal convection volume for improving patient outcomes in an international incident dialysis cohort treated with online hemodiafiltration. Kidney Int 2015;88:1108-1116.

19. Mostovaya IM, Bots ML, van den Dorpel MA, et al. Left ventricular mass in dialysis patients, determinants and relation with outcome: results from the Convective TRansport STudy (CONTRAST). PLoS One 2014;9:e84587.

20. Ahmadmehrabi S, Tang WH. Hemodialysis-induced cardiovascular disease. Semin Dial 2018;31:258-267.

21. Kawanishi $\mathrm{H}$. Is there enough evidence to prove that hemodiafiltration is superior? Blood Purif 2018;46:3-6.

22. Basile C, Davenport A, Blankestijn PJ. Why choose high volume online post-dilution hemodiafiltration? J Nephrol 2017;30:181186.

23. Blankestijn PJ, Grooteman MP, Nube MJ, Bots ML. Clinical evidence on haemodiafiltration. Nephrol Dial Transplant 2018;33 (suppl_3):iii53-iii58.

24. Locatelli F, Altieri P, Andrulli S, et al. Hemofiltration and hemodiafiltration reduce intradialytic hypotension in ESRD. J Am Soc Nephrol 2010;21:1798-1807.

25. Morena M, Jaussent A, Chalabi L, et al. Treatment tolerance and patient-reported outcomes favor online hemodiafiltration compared to high-flux hemodialysis in the elderly. Kidney Int 2017;91:1495-1509.

26. Chazot C, Deleuze S, Fadel B, et al. Is high-volume post-dilution haemodiafiltration associated with risk of fluid volume imbalance?: a national multicentre cross-sectional cohort study. Nephrol Dial Transplant 2019;34:2089-2095.

27. Donauer J, Schweiger C, Rumberger B, Krumme B, Böhler J. Reduction of hypotensive side effects during online-haemodiafiltration and low temperature haemodialysis. Nephrol Dial Transplant 2003;18:1616-1622.

28. Bellien J, Fréguin-Bouilland C, Joannidès R, et al. High-efficiency on-line haemodiafiltration improves conduit artery endothelial function compared with high-flux haemodialysis in end-stage renal disease patients. Nephrol Dial Transplant 2014;29:414-422.

29. Ağbaş A, Canpolat N, Çalışkan S, et al. Hemodiafiltration is associated with reduced inflammation, oxidative stress and improved endothelial risk profile compared to high-flux hemodialysis in children. PLoS One 2018;13:e0198320.

30. Shroff R, Smith C, Ranchin B, et al. Effects of hemodiafiltration versus conventional hemodialysis in children with ESKD: the 
HDF, Heart and Height Study. J Am Soc Nephrol 2019;30:678691.

31. den Hoedt CH, Bots ML, Grooteman MP, et al. Online hemodiafiltration reduces systemic inflammation compared to low-flux hemodialysis. Kidney Int 2014;86:423-432.

32. Charitaki E, Belman D, Davenport A. Treatment with haemodiafiltration stabilises vascular stiffness (measured by aortic pulse wave velocity) compared to haemodialysis. Nephron Clin Pract 2014;128:185-191.

33. Ohtake T, Oka M, Ishioka K, et al. Cardiovascular protective effects of on-line hemodiafiltration: comparison with conventional hemodialysis. Ther Apher Dial 2012;16:181-188.

34. Bargnoux AS, Klouche K, Fareh J, et al. Prohormone brain natriuretic peptide (proBNP), BNP and N-terminal-proBNP circulating levels in chronic hemodialysis patients: correlation with ventricular function, fluid removal and effect of hemodiafiltration. Clin Chem Lab Med 2008;46:1019-1024.

35. Czifra A, Páll A, Kulcsár J, et al. Hemodialysis and hemodiafiltration differently modulate left ventricular diastolic function. BMC Nephrol 2013;14:76.

36. Páll A, Czifra Á, Sebestyén V, et al. Hemodiafiltration and hemodialysis differently affect $\mathrm{P}$ wave duration and dispersion on the surface electrocardiogram. Int Urol Nephrol 2016;48:271-277.

37. Hameed I, Gaudino M, Naik A, et al. Comparison of the effects of hemodialysis and hemodiafiltration on left ventricular hypertrophy in end-stage renal disease patients: a systematic review and meta-analysis. Semin Dial 2020;33:120-126.

38. Park KW, Bae SK, Lee B, et al. The effect of on-line hemodiafiltration on heart rate variability in end-stage renal disease. Kidney Res Clin Pract 2013;32:127-133.

39. Locatelli F, Carfagna F, Del Vecchio L, La Milia V. Haemodialysis or haemodiafiltration: that is the question. Nephrol Dial Transplant 2018;33:1896-1904.

40. Bowry SK, Canaud B. Achieving high convective volumes in online hemodiafiltration. Blood Purif 2013;35 Suppl 1:23-28.

41. Mostovaya IM, Grooteman MP, Basile C, et al. High convection volume in online post-dilution haemodiafiltration: relevance, safety and costs. Clin Kidney J 2015;8:368-373.

42. Maduell F, Varas J, Ramos R, et al. Hemodiafiltration reduces allcause and cardiovascular mortality in incident hemodialysis patients: a propensity-matched cohort study. Am J Nephrol 2017;46:288-297.

43. Schiffl H. High-volume online haemodiafiltration treatment and outcome of end-stage renal disease patients: more than one mode. Int Urol Nephrol 2020;52:1501-1506.
44. Maduell F. Is there an 'optimal dose' of hemodiafiltration? Blood Purif2015;40 Suppl 1:17-23.

45. Ronco C. Hemodiafiltration: technical and clinical issues. Blood Purif 2015;40 Suppl 1:2-11.

46. Chapdelaine I, Mostovaya IM, Blankestijn PJ, et al. Treatment policy rather than patient characteristics determines convection volume in online post-dilution hemodiafiltration. Blood Purif 2014;37:229-237.

47. Chapdelaine I, de Roij van Zuijdewijn CL, Mostovaya IM, et al. Optimization of the convection volume in online post-dilution haemodiafiltration: practical and technical issues. Clin Kidney J 2015;8:191-198.

48. Penne EL, van der Weerd NC, Bots ML, et al. Patient- and treatment-related determinants of convective volume in post-dilution haemodiafiltration in clinical practice. Nephrol Dial Transplant 2009;24:3493-3499.

49. Maduell F, Ojeda R, Rodas L, et al. On-line haemodiafiltration with auto-substitution: assessment of blood flow changes on convective volume and efficiency. Nefrologia 2015;35:50-57.

50. Marcelli D, Kopperschmidt P, Bayh I, et al. Modifiable factors associated with achievement of high-volume post-dilution hemodiafiltration: results from an international study. Int J Artif Organs 2015;38:244-250.

51. Canaud B, Vienken J, Ash S, Ward RA; Kidney Health Initiative HDF Workgroup. Hemodiafiltration to address unmet medical needs ESKD patients. Clin J Am Soc Nephrol 2018;13:1435-1443.

52. Masakane I, Kikuchi K, Kawanishi H. Evidence for the clinical advantages of predilution on-line hemodiafiltration. Contrib Nephrol 2017;189:17-23.

53. Akizawa T, Koiwa F. Clinical expectation of online hemodiafiltration: a Japanese perspective. Blood Purif 2015;40 Suppl 1:12-16.

54. de Roij van Zuijdewijn CL, Chapdelaine I, Nubé MJ, et al. Achieving high convection volumes in postdilution online hemodiafiltration: a prospective multicenter study. Clin Kidney J 2017;10:804-812.

55. Maduell F, Broseta JJ, Gómez M, et al. Determining factors for hemodiafiltration to equal or exceed the performance of expanded hemodialysis. Artif Organs 2020;44:E448-E458.

56. Marcelli D, Scholz C, Ponce P, et al. High-volume postdilution hemodiafiltration is a feasible option in routine clinical practice. ArtifOrgans 2015;39:142-149.

57. van Loon MM, Kessels AG, van der Sande FM, Tordoir JH. Cannulation practice patterns in haemodialysis vascular access: predictors for unsuccessful cannulation. J Ren Care 2009;35:8289. 
58. National Kidney Foundation. Clinical update - needles and cannulas for arteriovenous fistula access: more options promote better outcomes [Internet]. New York: National Kidney Foundation; c2016 [cited 2021 Nov 14]. Available from: https://www. kidney.org/content/clinical-update-needles-and-cannulas-arteriovenus-fistula-access-more-options-promote-better.

59. Gauly A, Parisotto MT, Skinder A, et al. Vascular access cannulation in hemodialysis patients: a survey of current practice and its relation to dialysis dose. J Vasc Access 2011;12:358-364.

60. Hassell DR, van der Sande FM, Kooman JP, Tordoir JP, Leunissen KM. Optimizing dialysis dose by increasing blood flow rate in patients with reduced vascular-access flow rate. Am J Kidney Dis 2001;38:948-955.

61. Tattersall JE, Ward RA; EUDIAL group. Online haemodiafiltration: definition, dose quantification and safety revisited. Nephrol Dial Transplant 2013;28:542-550.

62. Teatini U, Steckiph D, Romei Longhena G. Evaluation of a new online hemodiafiltration mode with automated pressure control of convection. Blood Purif 2011;31:259-267.

63. Canaud B, Koehler K, Bowry S, Stuard S. What is the optimal target convective volume in on-line hemodiafiltration therapy? Contrib Nephrol 2017;189:9-16.

64. Jo YI, Kim KS, Chung KH, et al. High-volume postdilution online hemodiafiltration is possible even at low blood flow rate [Abstract]. J Am Soc Nephrol 2019;30:557.

65. Kim DH, Lee YK, Park HC, et al. Stepwise achievement of high convection volume in post-dilution hemodiafiltration: a prospective observational study. Semin Dial 2021;34:368-374.

66. Schiffl H. Online hemodiafiltration and mortality risk in endstage renal disease patients: a critical appraisal of current evidence. Kidney Res Clin Pract 2019;38:159-168.

67. Blankestijn PJ, Fischer KI, Barth C, et al. Benefits and harms of high-dose haemodiafiltration versus high-flux haemodialysis: the comparison of high-dose haemodiafiltration with high-flux haemodialysis (CONVINCE) trial protocol. BMJ Open 2020; 10:e033228. 